\title{
Cough and ventilatory adjustments evoked by aerosolised capsaicin and distilled water (fog) in man
}

\author{
Federico Lavorini ${ }^{a}$, Tito Pantaleo ${ }^{\mathrm{b}}$, Pietro Geri ${ }^{\mathrm{a}}$, Donatella Mutolo ${ }^{\mathrm{a}}$, \\ Massimo Pistolesi ${ }^{\text {a }}$, Giovanni A. Fontana ${ }^{\text {a,* }}$ \\ ${ }^{a}$ Dipartimento di Area Critica Medico Chirurgica, Unità Funzionale di Medicina Respiratoria, Università di Firenze, \\ Viale G.B. Morgagni 85, 50134 Firenze, Italy \\ ${ }^{\mathrm{b}}$ Dipartimento di Scienze Fisiologiche, Università di Firenze, Viale G.B. Morgagni 63, 50134 Firenze, Italy
}

Accepted 23 October 2006

\begin{abstract}
Airway receptors mediate cough and ventilatory adjustments. Simultaneous assessment of cough sensory-motor components and changes in breathing pattern may provide insights into the receptor(s) prevailingly stimulated by inhaled irritants. Nineteen subjects inhaled capsaicin and fog up to threshold concentrations for cough. Cough intensity, respiratory sensations and changes in breathing pattern induced by the two irritants were compared. Capsaicin and fog cough threshold values did not correlate. Coughing induced by both agents was preceded by qualitatively similar sensations and by significant increases in minute ventilation and respiratory drive due to selective increases in tidal volume $(P<0.01)$. Cough intensity was similar with both agents. Cough frequency and the intensity of the urge to cough were higher with capsaicin $(P<0.01)$. The lack of correlation between fog and capsaicin cough threshold values suggests differences in the neural mechanisms activated. The selective increase in tidal volume suggests prevailing involvement of rapidly adapting receptors. The stronger sensations evoked by capsaicin may contribute to the higher cough frequency observed with this agent.
\end{abstract}

(c) 2006 Elsevier B.V. All rights reserved.

Keywords: Airway receptors; Respiratory control; Respiratory sensations; Electromyography; Ultrasonically nebulised distilled water

\section{Introduction}

Cough is a vagally mediated respiratory defence reflex initiated by the activation of sensory fibres carried by the vagus nerves, with terminals primarily located in the proximal tract of the airways. At least two distinct types of airway sensory receptors, or sensors (Yu, 2005), have been implicated in cough mediation (Widdicombe, 2001 for review). One type is characterised as rapidly adapting receptors (RARs), with prominent mechanical and less pronounced chemical sensitivity; their role in cough mediation is widely recognised (Widdicombe, 2001). The other type is represented by the sensory endings of the non-myelinated C-fibres (C-fibre receptors); they have little mechanical sensitivity but are exquisitely responsive to endogenous and exogenous chemicals (Lee and Pisarri, 2001). The role of these sensory endings in cough mediation is still object of

\footnotetext{
* Corresponding author. Tel.: +39 055 413183; fax: +39055 4223202 .

E-mail address: g.fontana@ dac.unifi.it (G.A. Fontana).
}

debate (Widdicombe, 2001). Animal experiments performed in vivo have revealed that RARs and C-fibre sensory receptors may be activated by both non-isotonic water solutions and capsaicin, albeit to different extents (Pisarri et al., 1991, 1992; Mohammed et al., 1993; Morikawa et al., 1997), and many inhalation studies have confirmed the powerful tussigenic action of these agents in normal subjects and in patients with respiratory and nonrespiratory disorders (Higenbottam et al., 1989; Fontana et al., 1997, 1998, 1999a,b; Davenport et al., 2002; Kastelik et al., 2002; Dicpinigaitis, 2004). A peculiar type of low-threshold mechanoreceptors innervated by myelinated vagal fibres has been described recently in the guinea pig extrapulmonary airways (Canning et al., 2004; Mazzone, 2005; Mazzone et al., 2005). This sensor type has been suggested to represent the actual 'cough receptor' (Mazzone, 2005).

Afferent inputs originating from RARs and C-fibre receptors have been implicated also in the genesis of regulatory reflex responses, such as increases in respiratory drive and minute ventilation, but with distinctive modalities. Whilst the ventilatory response evoked by RAR activation mainly consists of 
hyperpnoea, i.e., an increase in tidal volume without consistent changes in respiratory rate (Coleridge and Coleridge, 1986; Yu et al., 1998; Sant'Ambrogio and Widdicombe, 2001), stimulation of C-fibre receptors causes rapid shallow breathing, i.e., an increase in respiratory rate associated with a reduction in the depth of breathing (Lee and Pisarri, 2001). We have recently shown that, in humans, coughing induced by fog inhalation is preceded by increases in respiratory drive and minute ventilation by way of selective increases in the volume component of the breathing pattern (Lavorini et al., 2001), pointing at the possibility that the sensors primarily stimulated were the RARs. To our knowledge, the ventilatory adjustments provoked by inhalation of tussigenic capsaicin concentrations in humans have not been assessed. In addition, little is known on the intensity of the cough motor responses evoked by this irritant. We have hypothesised that the assessment of cough and ventilatory responses provoked by capsaicin and fog inhalation in the same subjects could provide insights into the sensor type prevailingly stimulated by these irritants.

Recent studies performed on normal adult subjects have reported that a sense of an urge to cough occurs before a cough response is elicited by capsaicin inhalation (Davenport et al., 2002), a phenomenon that suggests processing of cough information by higher brain centres. In contrast, the respiratory sensations possibly associated with inhalation of tussigenic fog concentrations have not been described.

The present investigation was performed on normal subjects who inhaled capsaicin and fog concentrations at the threshold level for the cough reflex. The main purpose was to analyze and compare the cough motor responses and the ventilatory adjustments elicited by these irritants. An attempt was also made to investigate the concomitant respiratory sensations. In particular, we attempted at quantifying the intensity of the 'urge to cough' evoked by inhalation of threshold capsaicin and fog concentrations.

\section{Methods}

\subsection{Subjects}

We studied 19 healthy, non-smoker volunteers (14 males and 5 females, mean age 26.3 years; range, 19-33 years), who coughed in response to preliminary fog inhalation challenges. Subjects had not suffered from recent (less than 4 weeks) respiratory tract infections, and all had a normal baseline forced expiratory volume in $1 \mathrm{~s}\left(\mathrm{FEV}_{1}\right)$. All procedures were approved by the local ethics committee and adhered to the Recommendations of the Declaration of Helsinki for Human Experimentation. Individual informed consent was obtained after explanation of the procedures but not of the purpose of the study.

\subsection{Equipment and measurements}

Most of the equipment and measurements have already been described in details (Fontana et al., 1999b; Lavorini et al., 2001). During cough challenges, all participants breathed through a two-way, low-resistance shutter valve; the expiratory port of the valve was connected to a no. 4 Fleisch pneumotachograph and a flow transducer to record expiratory flow; the inspiratory port of the valve was in series with the ultrasonic nebuliser. The accuracy of the pneumotachograph-transducer assembly was assessed in preliminary trials (Petusevsky et al., 1980).

Maximal voluntary cough (MVC) efforts were obtained by encouraging each participant to cough as forcefully as possible. Reflex cough (RC) was induced by inhalation of either capsaicin or fog aerosols produced by a Mist-O ${ }_{2}-$ Gen EN143A ultrasonic nebuliser (Medical Equipment Services Inc., Fulton, IL, USA). The mass median aerodynamic diameter of aerosol particles generated by the nebuliser has been reported to be $5.7 \pm 1.4 \mu \mathrm{m}$ (Phipps and Gonda, 1990). For fog challenges, the nebuliser jar was filled with $180 \mathrm{ml}$ distilled water; the output could be adjusted by means of a potentiometer and monitored as a DC signal on an oscilloscope. The output could progressively be augmented in steps corresponding to $5 \%$ of the maximum attainable output level. The range of nebuliser outputs employed in the present experiments was from 30 to $100 \%$ of the maximum DC signal; the corresponding amount of nebulised water (mean values) ranged from 0.08 to $4.45 \mathrm{ml} / \mathrm{min}$ (Fontana et al., 1997). Cough threshold was taken as the lowest fog concentration capable of evoking at least one cough effort during two distinct challenges separated by a time interval of at least $30 \mathrm{~min}$ (Fontana et al., 1997).

A $10 \mathrm{mM}$ capsaicin stock solution was prepared (Dicpinigaitis, 2003) and subsequently diluted in physiological saline to obtain solutions ranging from 1.34 to $75.00 \mu \mathrm{M}$ (i.e., a $\sim 35 \%$ increase between concentrations). For capsaicin challenges, the nebuliser jar was initially filled with $180 \mathrm{ml}$ of the lowest capsaicin concentration increases in cough stimulus intensity were obtained by increasing the capsaicin concentration, whereas the nebuliser was always operated at $50 \%$ of the maximum attainable output.

Capsaicin or fog concentrations were alternated at random with physiological saline to avoid conditioned responses. Preliminary trials $(n=8)$ showed that, following inhalation of tussigenic capsaicin concentrations, cough may no longer be obtained with the same concentration for periods ranging from 30 to $180 \mathrm{~min}$. Therefore, the capsaicin cough threshold was taken as the lowest drug concentration capable of inducing at least one cough effort during a single challenge. Aerosolised capsaicin and fog were inhaled during resting tidal breathing and the inhalation time for each concentration was standardised at $1 \mathrm{~min} ; 2-3 \mathrm{~min}$ of rest were scheduled between concentrations. Possible changes in airway calibre induced by inhalation of these agents were monitored by $\mathrm{FEV}_{1}$ measurements.

As fully detailed elsewhere (Fontana et al., 1998, 1999b), the intensity of voluntary and reflex cough efforts was indexed in terms of the peak and rate of rise of the integrated electromyographic (IEMG) activity of the abdominal muscles, namely the obliquus externus muscle, as well as of the cough peak expiratory flow (PFC).

During inhalation of each capsaicin and fog concentration, participants were asked to rate their sensation of an urge to cough using a $10 \mathrm{~cm}$ long visual analogue scale continuously. The extremes of the sensation (i.e., 'no urge to cough at all' 
at point 0 , and 'extreme urge to cough' at point 10) were represented by the two ends of a visual display. They were also requested to slide a linear potentiometer to rate an increase or a decrease in the sensation, respectively.

The pattern of breathing was recorded by means of a calibrated (Sackner et al., 1989) respiratory inductive plethysmograph (Respitrace, Non-Invasive Monitoring System, Ardsley, NY, USA). From the sum of ribcage and abdominal signals we measured tidal volume $\left(V_{\mathrm{T}}\right)$ and the duration of inspiratory and expiratory times ( $T_{\mathrm{I}}$ and $T_{\mathrm{E}}$, respectively), and the total duration of the respiratory cycle $\left(T_{\mathrm{T}}\right)$; the respiratory frequency, the mean inspiratory flow $\left(V_{\mathrm{T}} / T_{\mathrm{I}}\right.$, an index of inspiratory drive), the duty cycle $\left(T_{\mathrm{I}} / T_{\mathrm{T}}\right.$, an index of respiratory timing), and the inspiratory minute ventilation $\left(\dot{V}_{\mathrm{I}}\right)$ were subsequently calculated. The partial pressure of end-tidal $\mathrm{CO}_{2}\left(P_{\mathrm{ET}_{\mathrm{CO}_{2}}}\right)$ was also continuously monitored (Normocap CD 102; Datex, Helsinki, Finland).

\subsection{Protocol}

Subjects attended the laboratory on two occasions separated by an interval of $\sim 48 \mathrm{~h}$ to be randomly challenged with either capsaicin or fog. Subjects were comfortably seated on a dentist's chair and were allowed to relax for 20-30 min. Then, participants performed 8-10 MVC manoeuvres, each separated by a 5-s interval, during which both the abdominal IEMG activity and the expiratory flow were recorded. Following a $\sim 10$-min recovery period, participants were connected to the nebuliser via the mouthpiece and the two-way valve, and were allowed to familiarise themselves with the equipment for further 5-6 min and reminded to breathe normally with as constant a pattern as possible. Following recording of the control breathing pattern (60-90 s), cough challenges were commenced. Upon completion of each challenge, subjects were asked to describe any respiratory sensation verbally; these were later transcribed from tape. Sensations were selected from a list based on our own experience and similar to that reported by Raji et al. (1995). Listed sensations included 'air hunger', 'difficulty with breathing', 'burning', 'irritation', 'choking', 'smoke in the throat', and 'any other sensation'. Following completion of the last scheduled cough challenge, subjects were requested to report which agent had provoked the more intense sensations.

Recordings of the studied variables could be displayed on a chart recorder (HP 7758 A, Hewlett-Packard, Paolo Alto, CA) and digitally acquired using a $\mathrm{PC}$ equipped with an $\mathrm{AD}$ interface (Digidata 1302A, Axon Instruments, Inc., Union City, CA) and appropriate software (Axoscope 8.1, Axon Instruments, Inc.).

\subsection{Data analysis}

We measured PFC in $1 / \mathrm{s}$ and the peak of IEMG activity (IEMGP) in arbitrary units during MVC and during fog and capsaicin challenges. For the purpose of this study, cough is defined as three-phase motor act characterised by an inspiratory effort, followed by a forced expiratory effort against a closed glottis followed by opening of the glottis and rapid expiratory airflow. In several circumstances, typically during capsaicin challenges, the cough motor pattern consisted of a single preparatory inspiration followed by multiple expiratory muscle discharges (see Fig. 2A). In such cases, multiple expiratory efforts preceded by a single inspiratory act were arbitrarily considered as a single cough, and only the first IEMGP was considered for subsequent analyses. Measurements of IEMG amplitudes were normalised by expressing them as a fraction of the highest IEMGP value recorded throughout each experimental session, which was consistently attained during MVC (Fontana et al., 1998). The normalised IEMGP values, expressed as relative units, were used for all subsequent analyses.

The duration of the rising phase of the abdominal IEMG activity during both $\mathrm{MVC}$ and $\mathrm{RC}$, i.e., the time duration of the expiratory IEMG ramp during cough, was termed TEC (Fontana et al., 1997; Lavorini et al., 2001). The ratio of IEMGP to TEC (IEMGP/TEC, an index of expiratory drive) was subsequently calculated for all considered cough efforts. As regards the MVC, the three manoeuvres with the highest PFC and IEMGP values were considered in each subject. Owing to the small variations in PFC and IEMG variables during both MVC and RC, average values were calculated in each subject and taken as single measurements for purpose of analysis.

As previously stated, cough threshold to fog, but not to capsaicin, was assessed during two separate challenges. Thus, cough frequency in fog studies was taken as the mean number of coughs recorded during the two 1-min inhalations of the threshold concentration; with capsaicin, cough frequency was taken as the number of coughs recorded during the 1-min inhalation of the threshold concentration. During each challenge, the time to onset, i.e., the time (in s) which elapsed between the beginning of inhalation of a tussigenic agent and the appearance of the first cough effort (Fontana et al., 1999a,b), was also calculated. The relationships between cough threshold values observed in fog and capsaicin studies were investigated by linear regression analysis. Linear regression analysis was used to examine the relationships between the intensity of the urge to cough and cough frequency. Comparisons between PFC, IEMGP, IEMGP/TEC, cough frequency and time to onset values recorded at threshold stimulus intensity during fog and capsaicin challenges were performed by using Wilcoxon's signed rank tests.

On both capsaicin and fog study days, respiratory variables were measured for an average of five consecutive breaths in the control periods and in the period preceding the appearance of cough. Mean values of control breathing pattern variables recorded prior to each of the two fog challenges were similar; they were therefore pooled and considered as a whole. Given the consistency of mean values of breathing pattern variables recorded during each of the two fog challenges prior to cough appearance, also these values were similarly treated. At threshold level for the cough reflex, changes in breathing pattern variables provoked by capsaicin and fog inhalation were compared by using Wilcoxon's signed rank tests. Changes in respiratory variables observed following cough cessation were obviously inconsistent and were not analyzed. Wilcoxon's signed rank tests were used to compare the intensity of the urge to cough recorded during inhalation of threshold capsaicin and fog 
concentrations. The same statistical procedure was used to compare data obtained in experiments aimed at rating the intensity of the urge to cough with those recorded in the same subjects during the main study. All reported values are means \pm S.D., unless otherwise stated; a $P$ value $<0.05$ was taken as significant.

\section{Results}

All subjects coughed in response to capsaicin and fog inhalation. The mean ( \pm S.D.) time to cough onset was $37.08 \pm 11.45 \mathrm{~s}$ with capsaicin and $33.45 \pm 12.27 \mathrm{~s}$ with fog; these values did not significantly differ. In all instances, coughing terminated within $15 \mathrm{~s}$ following stimulus cessation. Capsaicin cough threshold values ranged from 2.37 to $17.80 \mu \mathrm{M}$, and the corresponding median value was $11.05 \mu \mathrm{M}$. In fog challenges, cough threshold values ranged from 0.73 to $2.35 \mathrm{ml} / \mathrm{min}$ and the median value was $1.09 \mathrm{ml} / \mathrm{min}$. No correlation was found between individual cough threshold values observed in capsaicin and fog challenges (Fig. 1). During the 1-min inhalation of the threshold capsaicin concentration, cough frequency ranged from 2 to 12 (median value 8 ), whereas in fog challenges cough frequency ranged from 1 to 4 (median value 2). Cough frequency with capsaicin turned out to be significantly higher than with fog $(P<0.001)$. In no occasion did any of the subjects display a significant change in control $\mathrm{FEV}_{1}$ following capsaicin or fog inhalation.

Mean values of IEMG-related variables and PFC attained by the subjects during MVC on each study day were similar, as were those recorded during coughing provoked by inhalation

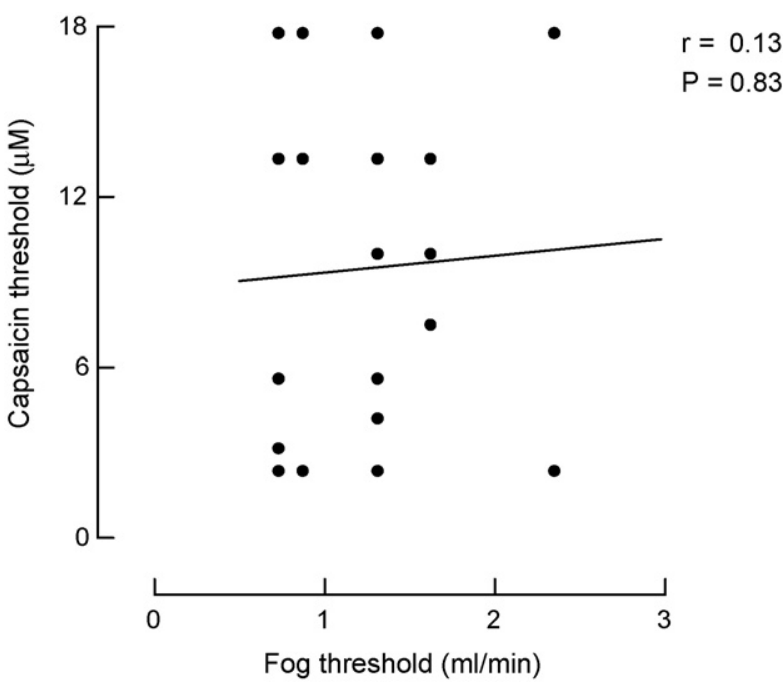

Fig. 1. Lack of relationship between individual cough threshold values observed during capsaicin and fog cough challenges. Data from 19 subjects.

of threshold fog and capsaicin concentrations (Table 1). With both tussigenic agents, the mean values of PFC and of abdominal IEMGP and IEMGP/TEC recorded during RC at threshold level attained approximately $80 \%$ of those recorded during MVC $(P<0.01)$.

In control conditions, mean values of breathing pattern variables recorded on each study day were similar (Table 2). Inhalation of sub-threshold fog and capsaicin concentrations caused only minimal and not significant changes in the pattern

Table 1

Mean values of variables of abdominal integrated electromyographic activity and of peak flow rates during coughing produced either voluntarily or by inhalation of threshold capsaicin and fog concentrations in 19 subjects

\begin{tabular}{lllll}
\hline & \multicolumn{2}{l}{ Capsaicin study day } & & Fog study day \\
\cline { 2 - 3 } & MVC & RC & MVC & $0.86 \pm 0.22$ \\
\hline IEMGP (RU) & $0.85 \pm 0.21$ & $0.76 \pm 0.20^{*}$ & $0.21 \pm 0.04$ & $0.75 \pm 0.16^{*}$ \\
TEC (s) & $0.22 \pm 0.06$ & $0.21 \pm 0.10$ & $4.51 \pm 1.32$ & $3.78 \pm 0.91^{*}$ \\
IEMGP/TEC (RU/s) & $4.34 \pm 1.92$ & $3.81 \pm 1.10^{*}$ & $6.13 \pm 0.60$ & $4.96 \pm 1.02^{*}$ \\
PFC (1/s) & $5.85 \pm 0.57$ & $4.94 \pm 0.44^{*}$ & & 6.06 \\
\hline
\end{tabular}

Values are means \pm S.D.; MVC, maximal voluntary cough; RC, reflex cough at threshold level; IEMGP, peak amplitude of the integrated abdominal electromyographic activity; TEC, time duration of the expiratory IEMG ramp; IEMGP/TEC, rate of rise of the IEMG activity; PFC, cough peak flow; RU, relative units. ${ }^{*} P<0.01$ compared with the corresponding MVC values (Wilcoxon signed rank tests). There was no significant difference between MVC and RC values recorded on capsaicin and fog study days.

Table 2

Comparisons of mean changes in values of breathing pattern variables recorded in control conditions (C) and during inhalation of threshold (T) fog and capsaicin concentrations

\begin{tabular}{|c|c|c|c|c|c|c|}
\hline & \multicolumn{3}{|c|}{ Capsaicin study day } & \multicolumn{3}{|l|}{ Fog study day } \\
\hline & $\mathrm{C}$ & $\mathrm{T}$ & $\Delta$ & $\mathrm{C}$ & $\mathrm{T}$ & $\Delta$ \\
\hline$V_{\mathrm{T}}(1)$ & $0.70 \pm 0.11$ & $0.86 \pm 0.14^{*}$ & $0.16 \pm 0.07$ & $0.68 \pm 0.11$ & $0.84 \pm 0.14^{*}$ & $0.16 \pm 0.05$ \\
\hline$T_{\mathrm{I}} / T_{\mathrm{T}}$ & $0.37 \pm 0.06$ & $0.35 \pm 0.06$ & $-0.01 \pm 0.04$ & $0.36 \pm 0.06$ & $0.36 \pm 0.06$ & $-0.10 \pm 0.13$ \\
\hline fR (breath/min) & $14.58 \pm 3.68$ & $14.91 \pm 3.49$ & $0.32 \pm 0.78$ & $14.40 \pm 3.55$ & $14.65 \pm 3.91$ & $0.25 \pm 0.76$ \\
\hline$\dot{V}_{\mathrm{I}}(\mathrm{l} / \mathrm{min})$ & $9.82 \pm 2.08$ & $12.13 \pm 2.93^{*}$ & $2.32 \pm 1.21$ & $9.68 \pm 2.03$ & $11.66 \pm 2.20^{*}$ & $1.98 \pm 0.44$ \\
\hline
\end{tabular}

Values are means \pm S.D.; $\Delta$, mean difference between $\mathrm{T}$ and $\mathrm{C} ; V_{\mathrm{T}}$, tidal volume; $T_{\mathrm{I}} / T_{\mathrm{T}}$, duty cycle; $V_{\mathrm{T}} / T_{\mathrm{I}}$, mean inspiratory flow; fR, respiratory frequency; $\dot{V}_{\mathrm{I}}$, inspiratory minute ventilation; $P_{\mathrm{ET}_{\mathrm{CO}_{2}}}$, partial pressure of end-tidal $\mathrm{CO}_{2} .{ }^{*} \mathrm{P}<0.01$ compared with control conditions (Wilcoxon signed rank tests). 
(A)

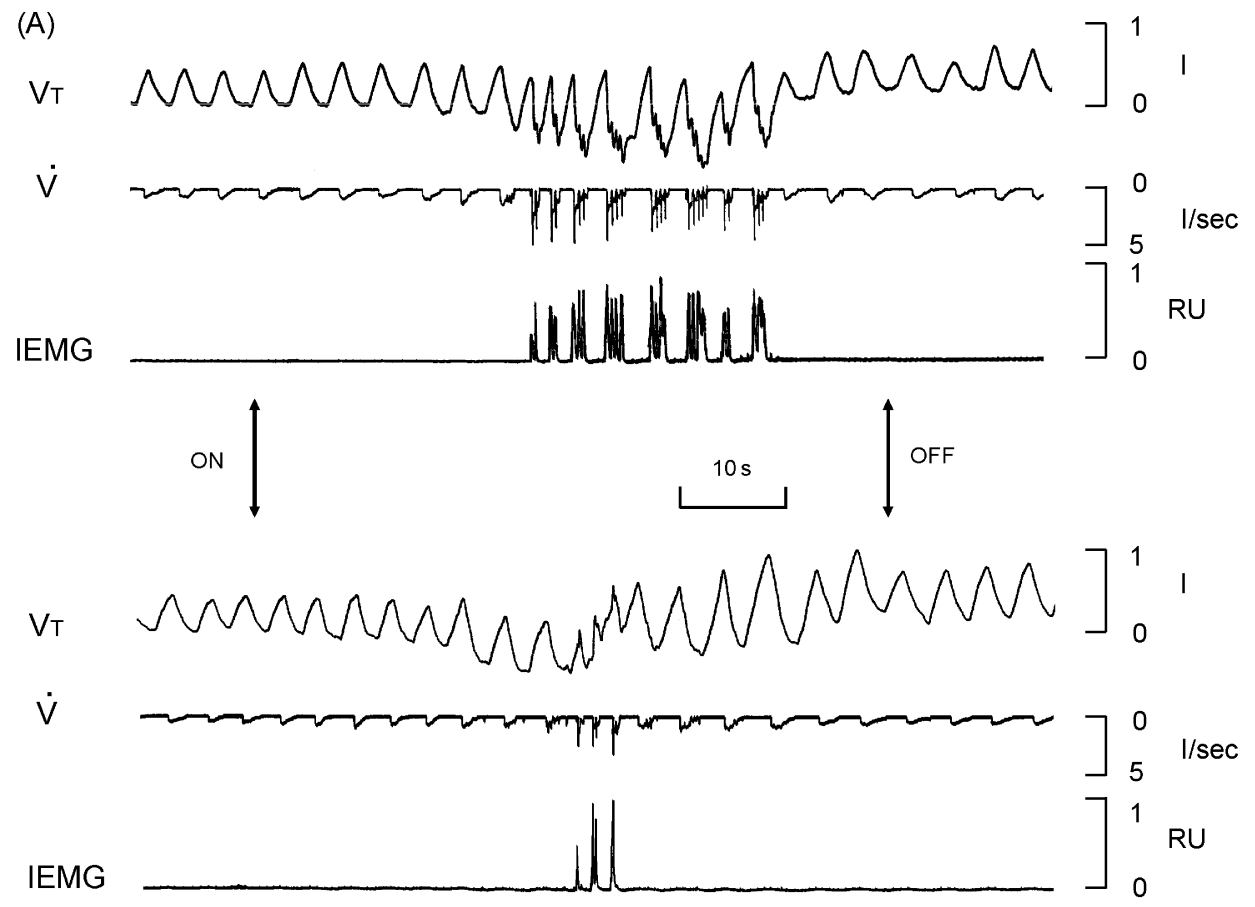

(B)

Fig. 2. Examples of cough responses and ventilatory adjustments induced by inhalation of capsaicin and fog at threshold level for the cough reflex in one subject. (A) Inhalation of capsaicin and (B) inhalation of fog. Arrows mark the beginning and end of the inhalation period. RU, relative units. Traces are $V_{\mathrm{T}}$, tidal volume; $\dot{V}$, expiratory flow; IEMG, integrated electromyographic activity of the abdominal muscles.

of breathing. Conversely, with both tussigenic agents (Fig. 2), coughing at threshold level was consistently preceded by significant increases in $\dot{V}_{\mathrm{I}}$ and $V_{\mathrm{T}} / T_{\mathrm{I}}$ that were due to a significant rise in $V_{\mathrm{T}}$ without appreciable changes in respiratory timing (Table 2). The ventilatory adjustments that preceded the onset of cough usually caused only slight, not significant reductions in $P_{\mathrm{ET}_{\mathrm{CO}}}$ (Table 2). Mean changes in the pattern of breathing during inhalation of threshold capsaicin and fog concentrations are also represented in Fig. 3 using conventional Hey plots (Hey et al., 1966).

In most subjects $(n=15)$, inhalation of near-threshold capsaicin and fog concentrations caused the appearance of the sensation of an urge to cough. At threshold level for the cough reflex, the sensation was perceived by all subjects; its mean intensity during capsaicin inhalation $(8.52 \pm 0.69 \mathrm{~cm})$ was slightly but significantly $(P<0.01)$ stronger than that perceived during fog inhalation $(7.17 \pm 0.59 \mathrm{~cm})$. For both capsaicin and fog, linear regression analysis showed a correlation between cough frequency and urge to cough values at threshold level (Fig. 4). Examples of the ventilatory adjustments, cough motor responses, and intensity of the urge to cough elicited by inhalation of threshold capsaicin and fog concentrations in a representative subject are illustrated in Fig. 5. In addition to the urge to cough, inhalation of threshold or near-threshold capsaicin and fog concentrations evoked other respiratory sensations in most of the subjects. Such sensations were perceived at the level of the throat or chest and were mainly described as 'irritation'; 'burning' or 'choking' sensations were less frequently reported. In no instance these sensations were powerful enough to prevent the prosecution of the trial. Subjects consistently reported verbally that sensations were qualitatively 'similar' with both tussigenic agents; however, sensations were judged to be 'stronger' with capsaicin in 17 subjects, and of 'more or less the same intensity' in the other 2 .

\section{Discussion}

The novel and interesting features of this research are the following. First, the appearance of cough induced by capsaicin inhalation is preceded by increases in $\dot{V}_{\mathrm{I}}$ and $V_{\mathrm{T}} / T_{\mathrm{I}}$ that are similar to those observed in the present and previous fog studies and are accounted for by selective increases in $V_{\mathrm{T}}$. Second, there is no correlation between fog and capsaicin cough threshold values. Third, as with fog (Fontana et al., 1997), the magnitude of cough motor responses to threshold capsaicin concentrations approximates $80 \%$ of that observed during MVC. Fourth, this study is the first to report on sensations evoked by threshold fog concentrations and to provide a qualitative and quantitative comparison of the respiratory sensations evoked by the two irritants. Finally, we demonstrate that, with both irritants, cough appearance is preceded by sensations whose intensity relates to cough frequency and is stronger with capsaicin.

Cough is a vagally mediated airway defence reflex and the most important tussigenic zones are located at the level of the trachea and main intrathoracic airways (e.g., Widdicombe, 2001). Although there is no dispute that mechanical and chemical stimuli applied to the larynx also cause cough, we believe that the reflex responses observed in this study are mainly due to activation of sensors located below the laryngeal level. In facts, a considerable amount of information points at the possibility 

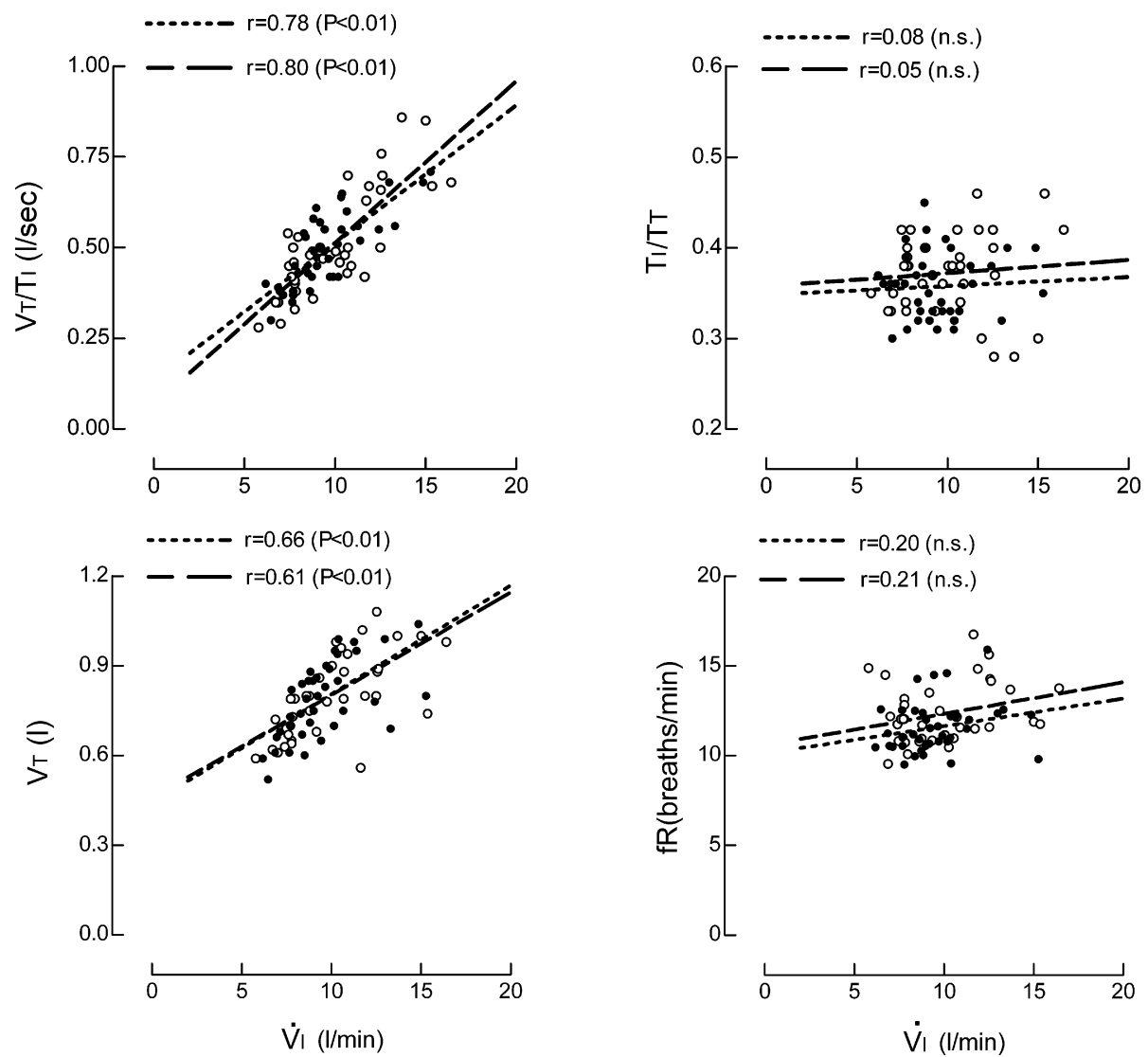

Fig. 3. Interrelationships between inspiratory minute ventilation $\left(\dot{V}_{\mathrm{I}}\right)$ and tidal volume $\left(V_{\mathrm{T}}\right)$, mean inspiratory flow $\left(V_{\mathrm{T}} / T_{\mathrm{I}}\right)$, the duty cycle $\left(T_{\mathrm{I}} / T_{\mathrm{T}}\right)$, and respiratory frequency (fR) illustrated by using conventional Hey plots. Data from 19 subjects. Note that the increases in $\dot{V}_{\mathrm{I}}$ correlate only with those in $V_{\mathrm{T}}$ and $V_{\mathrm{T}} / T_{\mathrm{I}}$.

that, in both animals and humans, the sensory endings mediating laryngeal cough are not promptly activated by agents such as fog and inhaled citric acid (Higenbottam et al., 1989; Forsberg et al., 1990; Stockwell et al., 1993; Tatar et al., 1996; Fontana et al., 1999b). This may be particularly true with threshold concentrations of irritants. The participation of laryngeal sensors

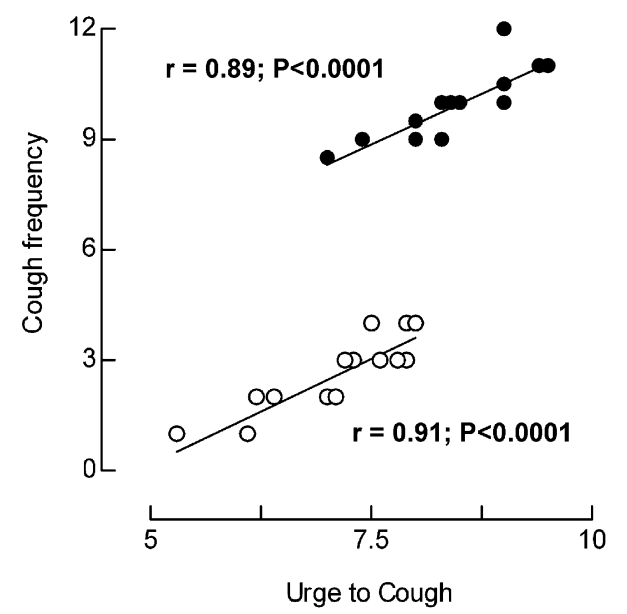

Fig. 4. Linear relationships between the intensity of the urge to cough and the number of cough efforts (cough frequency) recorded during 1-min inhalation of capsaicin and fog concentrations at threshold level for the cough reflex. Filled circles, capsaicin inhalation $(n=19)$; empty circles, fog inhalation $(n=19)$. Some data points overlap. in coughing induced by capsaicin inhalation in humans has not been investigated specifically.

As for the airway sensors responsible for the cough reflex, the RARs have been considered the most likely candidates (Coleridge and Coleridge, 1986; Widdicombe, 1996, 2001; Mazzone, 2005). The C-fibre receptors have also been implicated (Lee and Pisarri, 2001; Widdicombe, 2001). Recently, a peculiar type of low-threshold mechanoreceptors has been described in the guinea pig extrapulmonary airways and has been suggested to be the actual cough receptor (Canning et al., 2004; Mazzone, 2005; Mazzone et al., 2005). Although several in vivo experiments (Pisarri et al., 1991, 1992; Mohammed et al., 1993) indicate that in many animal species both the airway RARs and C-fibre receptors are activated by non-isosmolar solutions and capsaicin, a direct involvement of the latter sensor type in cough mediation is controversial (e.g., Widdicombe, 2001; Lee and Pisarri, 2001). Indeed, recent lines of evidence suggest a modifying and amplifying role for capsaicin-sensitive nerves in cough and other defensive reflexes in awake animals (Canning et al., 2006). Interestingly, it has been proposed that sensory nerve activation by inhaled irritants could also result in 'sensitisation' of central excitatory pathways regulating the cough reflex (Canning et al., 2004; Mazzone et al., 2005). Admittedly, present knowledge on the role played by the various types of airway sensors in the mediation of the observed respiratory responses is scanty, and it can only be speculated in our experimental conditions. However, it seems logical to 


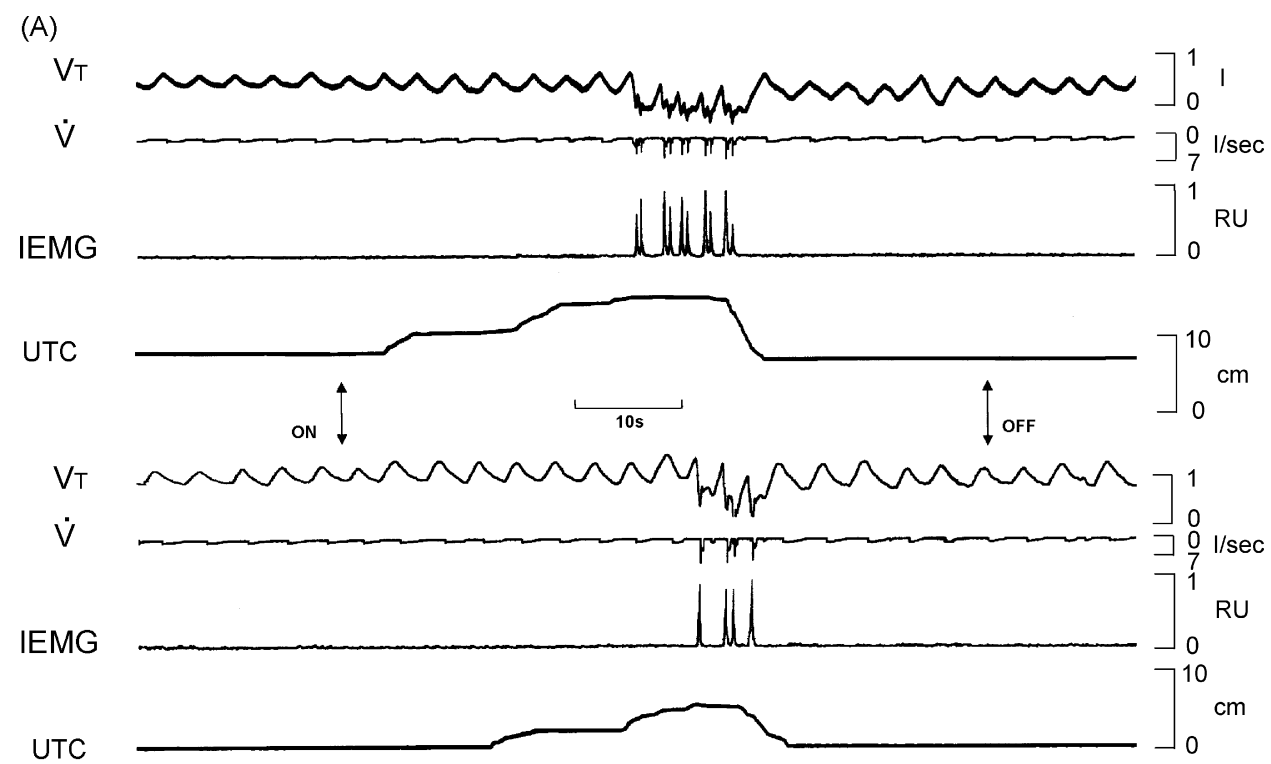

(B)

Fig. 5. Recordings of the urge to cough during capsaicin and fog challenges at threshold level for the cough reflex in one subject. (A) Inhalation of capsaicin and (B) inhalation of fog. Arrows mark the beginning and end of the inhalation period. RU, relative units. Traces are $V_{\mathrm{T}}$, tidal volume; $\dot{V}$, expiratory flow; IEMG, integrated electromyographic activity of the abdominal muscles; UTC, intensity of the urge to cough.

hypothesise that the lack of correlation between fog and capsaicin threshold values reflects different degrees of activation of the different sensors implicated in cough mediation, as well as different levels of central facilitatory interactions.

Airway sensors are also involved in the genesis of regulatory responses such as changes in ventilation and shaping of the breathing pattern (Coleridge and Coleridge, 1986). In the latter instances the role of the different airway sensor types, specifically that of RARs and C-fibre receptors, appears to be more precisely defined than in cough. In this connection, however, it should be borne in mind that virtually all information regarding reflex ventilatory adjustments provoked by airway irritants originates from animal experiments, and therefore it may be influenced profoundly by the anaesthesia.

It is generally agreed that the ventilatory reflex response evoked by C-fibre stimulation is the tachypnoea, a phenomenon also termed "rapid shallow breathing" (Lee and Pisarri, 2001). If the strength of C-fibre stimulation is intense enough, the tachypnoea may be preceded by a period of complete apnoea of variable duration (Karczewski and Widdicombe, 1969; Paintal, 1969, 1973; Coleridge and Coleridge, 1986; Tatar et al., 1988; Kay and Armstrong, 1990; see also Lee and Pisarri, 2001). On the other hand, the ventilatory effects produced by RARs stimulation mainly consist of hyperpnoea (Coleridge and Coleridge, 1986). The putative specific cough receptors described recently in the guinea pig, if present in humans, could be excited by fog and, indirectly, by capsaicin (Canning et al., 2004; Mazzone, 2004, 2005; Mazzone et al., 2005). Unfortunately, no information is available on the reflex ventilatory effects provoked by its activation.

With these limitations in mind, we attempted to get insights into the sensor type(s) prevailingly stimulated by fog and capsaicin by evaluating the changes in the pattern of breathing possibly associated with the inhalation of these irritants in awake humans. With no exception, such changes were characterised by increases in $V_{\mathrm{T}}$ and $V_{\mathrm{T}} / T_{\mathrm{I}}$ with minimal changes, if any, in respiratory timing (see Table 2, Figs. 2, 3 and 5). Thus, the present findings are in keeping with the possibility that both fog and capsaicin prevailingly activated RARs, either directly or indirectly (Widdicombe, 2003). Interestingly, it may be speculated that the increases in $V_{\mathrm{T}}$ preceding the appearance of cough (see Section 3 and Fig. 2) represent 'incomplete' attempts to cough; perhaps, when the $V_{\mathrm{T}}$ becomes large enough, it will trigger the next phases of cough. The fact that during inhalation of sub-threshold capsaicin and fog concentrations subjects often reported respiratory sensations, but no change in ventilation, is in keeping with the possibility that sensations and changes in ventilation provoked by inhaled irritants have different time courses.

We have shown that values of abdominal IEMGP, IEMGP/TEC and PFC during reflex cough evoked by inhalation of both capsaicin and fog were similar and approximated $80 \%$ of those recorded during MVC (see Table 1). The intensity of cough motor responses evoked by threshold capsaicin concentrations had not been assessed previously. Conceivably, with both agents, activation of the cough reflex recruits a considerable portion of the expulsive mechanism already at threshold level, thus allowing the achievement of expiratory flow velocities high enough to warrant effective airway clearing or removal of inhaled foreign bodies (Fontana, 2003; Fontana and Lavorini, 2006), thus providing a substantial safety factor. In real life, further needs of airway clearing may be coped with by small, additional recruitment of motor units in the contracting muscles and, perhaps more efficiently, by increasing the number of cough efforts (Fontana et al., 1997).

An interesting finding of the present study is that cough frequency was much higher with capsaicin than with fog. Several 
factors may account for the discrepancy. First, the phenomenon could be due to a steeper stimulus/response relationship for capsaicin than fog. Concentration- or dose-dependency of cough frequency has repeatedly been demonstrated for capsaicin (e.g., Midgren et al., 1992) but no data are available for fog. Since the present experiments were designed to investigate specifically the reflex respiratory responses evoked by threshold capsaicin and fog concentrations, they cannot provide information on the stimulus/response relationships for these tussigenic agents. Nonetheless, the fact that the cough time to onset turned out to be similar with both agents points at the possibility that steepness of the stimulus response/relationship is not a key factor in determining differences between capsaicin and fog cough frequency values. A second possible explanation relates with the differences in the mechanisms by which inhaled capsaicin and non-isosmolar solutions may activate cough-related sensory endings (Kollarik and Undem, 2006 for review), with capsaicin possibly exerting a longer lasting stimulation than fog and provoking a higher number of cough efforts. Lastly, another determinant of the higher cough frequency observed with capsaicin could be the stronger respiratory sensations evoked by this irritant. It is well documented that humans are able to sense and evaluate respiratory changes such as those induced by respiratory loads, bronchoconstriction, lung volume, airway pressure, changes in ventilation and blood gas tensions (see Davenport et al., 2002), and by i.v. administrations of chemicals (Raji et al., 1995; Butler et al., 2001). However, little is known on sensations evoked by inhalation of tussigenic agents. Recently, it has been reported (Davenport et al., 2002) that a sense of an urge to cough occurs before cough is produced in normal adults challenged with capsaicin. Furthermore, the intensity of the sensation closely correlates with both the capsaicin concentrations and the number of elicited thrusts (Davenport et al., 2002). In the present study, inhalation of threshold or near-threshold fog and capsaicin concentrations evoked a variety of respiratory sensations, including the urge to cough, that were qualitatively similar with both agents. In addition, at threshold level, the intensity of the urge to cough was stronger with capsaicin than with fog, and the intensity of the sensation correlates with the cough frequency, in agreement with previous findings (Davenport et al., 2002). It seems plausible, therefore, to hypothesise that the higher number of coughs evoked by capsaicin is also due to the stronger respiratory sensations, particularly the urge-to-cough. In this context, it can be recalled that putatively selective stimulants of the bronchopulmonary C-fibres have consistently failed to evoke cough in anaesthetised animals such as cats, dogs and guinea pigs, and may actually inhibit it (reviewed in Mazzone et al., 2004). Therefore, it has been proposed that C-fibre activation results in the conscious perception of airway irritation and in respiratory sensations ultimately leading to cough (Mazzone et al., 2004). Accordingly, human subjects can voluntarily suppress capsaicin-induced cough at least up to a certain stimulus intensity (Hutchings et al., 1993). Nevertheless, we are confident that the observed cough responses and differences in cough frequency do not entirely depend upon supramedullary influences, since prior to each trial subjects were carefully instructed to avoid any attempt to facilitate or inhibit cough, that is, they were recommended to cough freely when they could not abstain from doing so. Cough frequency and intensity of respiratory sensations were higher with capsaicin than fog; therefore, it may be that capsaicin is more effective in stimulating airway sensors, either RAR, C-fibres, or other nociceptors (Canning et al., 2004; Mazzone et al., 2005). Noticeably, in agreement with the results obtained by Davenport et al. (2002), both the urge to cough and the increases in $\dot{V}_{\text {I }}$ precede the appearance of cough in the present study. This provides a correlative evidence that both phenomena are a direct consequence of airway receptor stimulation. The sensation evoked by tussigenic concentrations of irritants other than capsaicin and fog remains to be assessed.

In conclusion, the pattern of the ventilatory responses points at a prevailing activation of the RARs with both tussigenic agents. The relationships between airway receptor stimulation, respiratory sensations and respiratory reflex responses are still poorly understood. Speculatively, capsaicin and fog could excite airway receptors that trigger a neural processes leading, with different time courses, to hyperpnoea, respiratory sensations and cough. It is also conceivable that airway receptor stimulation could first provoke respiratory sensations; when these become strong enough, changes ventilation ensue. Respiratory sensations may also facilitate cough, possibly via a cortical mechanism. Further investigations are needed to clarify the complex features of coughing, respiratory sensations and ventilatory adjustments evoked by inhaled irritants.

\section{Acknowledgements}

This work was supported by grants from the Ministero dell'Istruzione, dell'Università e della Ricerca of Italy.

\section{References}

Butler, J.E., Anand, A., Crawford, M.R., Glanville, A.R., McKenzie, D.K., Paintal, A.S., Taylor, J.L., Gandevia, S.C., 2001. Changes in respiratory sensations induced by lobeline after human bilateral lung transplantation. J. Physiol. 534, 583-593.

Canning, B.J., Mazzone, S.B., Meeker, S.N., Mori, N., Reynolds, S.M., Undem, B.J., 2004. Identification of the tracheal and laryngeal afferent neurones mediating cough in anaesthetized guinea-pigs. J. Physiol. 557, 543-558.

Canning, B.J., Farmer, G., Mori, N., 2006. Mechanistic studies of acid-evoked coughing in anesthetized guinea pigs. Am. J. Physiol. 291, R454-R463.

Coleridge, H.M., Coleridge, J.C.G., 1986. Reflexes evoked from tracheobronchial tree and lungs. In: Handbook of Physiology, Section 3: The Respiratory System, vol. III: Control of Breathing, Part 1. American Physiological Society, Washington, DC, pp. 395-429.

Davenport, P.W., Sapienza, C.M., Bolser, D.C., 2002. Psychophysical assessment of the urge-to-cough. Eur. Respir. Rev. 12, 249-253.

Dicpinigaitis, P.V., 2003. Cough reflex sensitivity in cigarette smokers. Chest $123,685-688$

Dicpinigaitis, P.V., 2004. Cough. 4. Cough in asthma and eosinophilic bronchitis. Thorax 59, 71-72.

Fontana, G.A., Pantaleo, T., Lavorini, F., Boddi, V., Panuccio, P., 1997. A noninvasive electromyographic study on threshold and intensity of cough in humans. Eur. Respir. J. 10, 983-989.

Fontana, G.A., Pantaleo, T., Lavorini, F., Benvenuti, F., Gangemi, S., 1998 Defective motor control of coughing in Parkinson's disease. Am. J. Respir. Crit. Care Med. 158, 458-464. 
Fontana, G.A., Pantaleo, T., Lavorini, F., Maluccio, N.M., Mutolo, D., Pistolesi, M., 1999a. Repeatability of cough-related variables during fog challenges at threshold and suprathreshold stimulus intensity in humans. Eur. Respir. J. $13,1447-1450$.

Fontana, G.A., Pantaleo, T., Lavorini, F., Polli, G., Pistolesi, M., 1999b. Coughing in laryngectomized patients. Am. J. Respir. Crit. Care Med. 160, $1578-1584$.

Fontana, G.A., 2003. Motor mechanisms and the mechanics of cough. In: Chung, F., Widdicombe, J., Bushey, H. (Eds.), Cough: Causes, Mechanisms and Therapy. Blackwell Publishing, Oxford, UK, pp. 193-205.

Fontana, G.A., Lavorini, F., 2006. Cough motor mechanisms. Respir. Physiol. Neurobiol. 152, 266-281.

Forsberg, K., Karlsson, J.-A., Lundberg, J.M., Zackrisson, C., 1990. Effect of partial laryngeal denervation on irritant-induced cough and bronchoconstriction in conscious guinea pigs. J. Physiol. 422, 34.

Hey, E.N., Lloyd, B.B., Cunningham, D.J., Jukes, M.G., Bolton, D.P., 1966. Effects of various respiratory stimuli on the depth and frequency of breathing in man. Respir. Physiol. 1, 193-205.

Higenbottam, T., Jackson, T.M., Woolman, P., Lowry, R., Wallwork, J., 1989. The cough response to ultrasonically nebulized distilled water in heart-lung transplantation patients. Am. Rev. Respir. Dis. 140, 58-61.

Hutchings, H.A., Morris, S., Eccles, R., Jawad, M.S., 1993. Voluntary suppression of cough induced by inhalation of capsaicin in healthy volunteers. Respir. Med. 87, 379-382.

Karczewski, W., Widdicombe, J.G., 1969. The role of the vagus nerves in the respiratory and circulatory responses to intravenous histamine and phenyl diguanide in rabbits. J. Physiol. 201, 271-291.

Kastelik, J.A., Thompson, R.H., Aziz, I., Ojoo, J.C., Redington, A.E., Morice, A.H., 2002. Sex-related differences in cough reflex sensitivity in patients with chronic cough. Am. J. Respir. Crit. Care Med. 166, 961-964.

Kay, I.S., Armstrong, D.J., 1990. Phenylbiguanide not phenyldiguanide is used to evoke the pulmonary chemoreflex in anaesthetized rabbits. Exp. Physiol. 75, 383-389.

Kollarik, M., Undem, B.J., 2006. Sensory transduction in cough-associated nerves. Respir. Physiol. Neurobiol. 152, 243-254.

Lavorini, F., Fontana, G.A., Pantaleo, T., Camiciottoli, G., Castellani, W., Maluccio, N.M., Pistolesi, M., 2001. Fog-induced respiratory responses are attenuated by nedocromil sodium in humans. Am. J. Respir. Crit. Care Med. $163,117-1120$.

Lee, L.-Y., Pisarri, T., 2001. Afferent properties and reflex functions of bronchopulmonary C-fibers. Respir. Physiol. Neurobiol. 125, 3-15.

Mazzone, S.B., 2004. Sensory regulation of the cough reflex. Pulm. Pharmacol. Ther. 17, 361-368.

Mazzone, S.B., 2005. An overview of the sensory receptors regulating cough Cough $1,2$.

Mazzone, S.B., Mori, N., Canning, B.J., 2005. Synergistic interactions between airway afferent nerve subtypes regulating the cough reflex in guinea-pigs. J. Physiol. 569, 559-573.
Midgren, B., Hanson, L., Karlsson, J.-A., 1992. Capsaicin-induced cough in humans. Am. Rev. Respir. Dis. 146, 347-351.

Mohammed, S.P., Higenbottam, T.W., Adcock, J.J., 1993. Effects of aerosolapplied capsaicin, histamine and prostaglandin E2 on airway sensory receptors of anaesthetized cats. J. Physiol. 469, 51-66.

Morikawa, T., Gallico, L., Widdicombe, J., 1997. Actions of moguisteine on cough and pulmonary rapidly adapting receptor activity in the guinea pig. Pharmacol. Res. 35, 113-118.

Paintal, A.S., 1969. Mechanism of stimulation of type J pulmonary receptors. J. Physiol. 203, 511-532.

Paintal, A.S., 1973. Vagal sensory receptors and their reflex effects. Physiol. Rev. 53, 159-227.

Petusevsky, M.L., Lyons, L.D., Smith, A.A., Epler, G.R., Gaensler, E.A., 1980. Calibration of time derivatives of forced vital capacity by explosive decompression. Am. Rev. Respir. Dis. 121, 343-350.

Phipps, P.R., Gonda, I., 1990. Droplets produced by medical nebulizers. Some factors affecting their size and solute concentration. Chest 97, 1327-1332.

Pisarri, T.E., Jonzon, A., Coleridge, H.M., Coleridge, J.C.G., 1991. Intravenous injection of hypertonic $\mathrm{NaCl}$ solution stimulates pulmonary $\mathrm{C}$-fibres in dogs. Am. J. Physiol. 260, H1522-H1530.

Pisarri, T.E., Jonzon, A., Coleridge, H.M., Coleridge, J.C.G., 1992. Vagal afferents and reflex responses to changes in surface osmolarity in lower airways of dogs. J. Appl. Physiol. 73, 2305-2313.

Raji, H., Singh, V.K., Anand, A., Paintal, A.S., 1995. Sensory origin of lobelineinduced sensations: a correlative study in man and cat. J. Physiol. 482, $235-246$

Sackner, M.A., Watson, H., Belsito, A.S., Feinerman, D., Suarez, M., Gonzalez, G., Bizousky, F., Krieger, B., 1989. Calibration of respiratory inductive plethysmography during natural breathing. J. Appl. Physiol. 66, 410-420.

Sant'Ambrogio, G., Widdicombe, J., 2001. Reflexes from airway rapidly adapting receptors. Respir. Physiol. 125, 33-45.

Stockwell, M., Lang, S., Zintel, T., White, C., Gallagher, G., 1993. Lack of importance of the superior laryngeal nerves in citric acid cough in humans. J. Appl. Physiol. 75, 613-617.

Tatar, M., Webber, S.E., Widdicombe, J.G., 1988. Lung C-fibre receptor activation and defensive reflexes in anaesthetized cats. J. Physiol. 402, 411-420.

Tatar, M., Karcolova, D., Pecova, R., Brozmanova, M., 1996. The role of partial laryngeal denervation on the cough reflex in awake guinea-pigs, rats and rabbits. Pulm. Pharmacol. 9, 371-372.

Widdicombe, J.G., 1996. Sensory neurophysiology of the cough reflex. J. Allergy Clin. Immunol. 98, S84-S90.

Widdicombe, J., 2001. Airway receptors. Respir. Physiol. Neurobiol. 125, 47-65.

Widdicombe, J.G., 2003. Overview of neural pathways in allergy and asthma. Pulm. Pharmacol. Ther. 16, 23-30.

Yu, J., 2005. Airway mechanosensors. Respir. Physiol. Neurobiol. 148, 217-243.

Yu, J., Zhang, J.F., Fletcher, E.C., 1998. Stimulation of breathing by activation of pulmonary peripheral afferents in rabbits. J. Appl. Physiol. 85, 1485-1492. 